\title{
BMJ Open Psychological correlates and binge drinking behaviours among Canadian youth: a cross-sectional analysis of the mental health pilot data from the COMPASS study
}

\author{
Alexandra Butler, ${ }^{\oplus 1}$ Isabella Romano, ${ }^{1}$ Karen Patte, ${ }^{2}$ Mark A Ferro, ${ }^{1}$ \\ Margaret de Groh, ${ }^{3}$ Ying Jiang, ${ }^{3}$ Scott T Leatherdale ${ }^{1}$
}

To cite: Butler A, Romano I, Patte K, et al. Psychological correlates and binge drinking behaviours among Canadian youth: a cross-sectional analysis of the mental health pilot data from the COMPASS study. BMJ Open 2019;9:e028558. doi:10.1136/ bmjopen-2018-028558

- Prepublication history for this paper is available online. To view please visit the journal online (http://dx.doi.org/10. 1136/bmjopen-2018-028558).

Received 13 December 2018 Revised 28 May 2019 Accepted 29 May 2019

Check for updates

(c) Author(s) (or their employer(s)) 2019. Re-use permitted under CC BY-NC. No commercial re-use. See rights and permissions. Published by BMJ.

${ }^{1}$ School of Public Health and Health Systems, University of Waterloo, Waterloo, Ontario, Canada

${ }^{2}$ Department of Health Sciences, Brock University, St Catharines, Ontario, Canada

${ }^{3}$ Applied Research Division, Public Health Agency of Canada, Ottawa, Ontario, Canada

Correspondence to

Alexandra Butler;

alle.butler@uwaterloo.ca

\section{ABSTRACT}

Objective The objective of this study was to examine associations between depression, anxiety and binge drinking among a large sample of Canadian youth, while testing the moderating effect of flourishing. This research uses data from the Cannabis, Obesity, Mental health, Physical activity, Alcohol, Smoking, Sedentary Behaviour (COMPASS) study (2012-2021) with a large sample size collecting data on youth health behaviours within Canadian secondary schools.

Design Cross-sectional

Setting 14 secondary schools across Ontario and British Columbia, Canada.

Participants A sample of grade 9-12 students $(n=6570)$ who participated in the Mental Health pilot of the COMPASS study

Primary and secondary outcome measures Selfreported questionnaires assessed student binge drinking behaviours ( $5 \geq$ drinks), symptoms of depression (Center for Epidemiologic Studies Depression Scale (Revised)-10 scores $\geq 10$ ) and anxiety (Generalised Anxiety Disorder 7-item Scale scores $\geq 10$ ), and flourishing (Diener's Flourishing Scale: 8-40).

Results In our sample of 6570 students, $37.0 \%$ of students reported binge drinking in the last year, and $41.4 \%$ and $31.7 \%$ of students report clinically-relevant symptoms of depression and anxiety, respectively. Anxiety (adjusted OR (AOR): 0.57, (99\% Cl 0.15 to 2.22)) and depression (AOR: $1.98,(99 \% \mathrm{Cl} 0.76$ to 5.13$)$ ) symptoms were not found to be associated with binge drinking and we did not detect any moderating role of flourishing. Rather, factors that were associated with increased odds of binge drinking included sports team participation (AOR: $1.67,(99 \% \mathrm{Cl} 1.37$ to 2.03$))$ and use of other substances (tobacco (AOR: 3.00, $(99 \% \mathrm{Cl} 2.12$ to 4.25$)$ ) and cannabis (AOR: $7.76,(99 \% \mathrm{Cl} 6.36$ to 9.46$)))$. Similar associations were found for frequency of binge drinking. Conclusions Consistent with existing literature, binge drinking behaviours were problematic, as well as clinically-relevant symptoms of depression and anxiety. However, mental health problems and well-being may not be responsible for explaining patterns of binge drinking in youth. Targeted intervention efforts towards student

\section{Strengths and limitations of this study}

- The COMPASS (Cannabis, Obesity, Mental health, Physical activity, Alcohol, Smoking, Sedentary Behaviour) project is a unique data system that provides an ideal platform to evaluate multiple co-occurring behavioural trajectories among a large sample of Canadian youth.

- Only cross-sectional results are reported as this study used first year pilot data for a mental health module to examine associations between mental health problems and binge drinking; future waves of COMPASS will allow mental health trajectories to be explored.

- Purposive sampling methods were used and results may not be representative of all Ontario and British Columbia secondary schools.

- Although findings may be subject to reporting or recall bias as data were self-reported, passive permission protocols and unique self-generated identification codes have been implemented to preserve anonymity.

- This study was unable to examine broader social environments (eg, social acceptability of alcohol, sport team dynamics) that may be associated with alcohol consumption patterns.

athletes and concurrent substance users are necessary for addressing binge drinking in youth populations.

\section{INTRODUCTION}

Although federal and provincial legislations in Canada prohibit alcohol consumption for those under the age of 18 or 19 , about $25 \%$ of Canadian students in grade 7-12 report pastyear binge drinking, which has been previously operationalised as consuming five or more drinks on one occasion. ${ }^{12}$ In comparison, $17 \%$ of youth report past-year cannabis use and $10 \%$ report using tobacco products 
in the past 30 days. $^{2}$ Acute and chronic health risks of early-onset alcohol use can be severe, including potential neurological and psychosocial concerns. As brain development continues throughout adolescence, youth are especially vulnerable to the effects of alcohol, particularly in high volumes and frequent doses. ${ }^{34}$ Alcohol consumption during adolescence may contribute to future experiences of psychiatric and substance use disorders, ${ }^{35}$ and youth who partake in binge drinking are at increased risk for such adverse health outcomes compared with youth who do not. ${ }^{6-9}$ Binge drinking ${ }^{10}{ }^{11}$ and frequency of drunkenness ${ }^{12}$ during adolescence have been linked to negative psychological outcomes including conduct disorder, anxiety, depression and suicidal behaviour.

Binge drinking has many negative physiological effects on the brain $^{13}$ and may lead to impaired mood and symptoms of depression among youth. ${ }^{14}{ }^{15}$ Longitudinal research has found problem-use of alcohol during adolescence to be predictive of depression in young adulthood. ${ }^{14}$ Alternatively, depression has been found to predict subsequent alcohol use, and research suggests that youth who experience depressive symptoms may binge drink in an attempt to reduce negative affect. ${ }^{16}{ }^{17}$ Negative affect is a strong predictor of harmful drinking and may distinguish between customary or social drinking behaviours and abusive drinking trajectories. ${ }^{18}$ Males and females demonstrate unique vulnerabilities to binge drinking in relation to biopsychosocial functioning. ${ }^{19}$ However, consequences from binge drinking may be more severe or prevalent in females considering they are at an increased risk of experiencing symptoms of mental health problems. ${ }^{19}$ Although adolescent males have previously been at greater risk for alcohol use and use at higher frequencies, this gender gap has gradually lessened where binge drinking among adolescent females has become more common. ${ }^{20}{ }^{21}$ Previous studies have cited risk-taking behaviour ${ }^{22}$ including tobacco and cannabis use, ${ }^{23}$ participation in team sports, ${ }^{2425}$ being of white ethnicity ${ }^{2526}$ and having higher amounts of spending money ${ }^{25}$ as common risk factors for binge drinking among secondary school students.

Widespread alcohol use among youth may also be explained by the Theory of Planned Behaviour. ${ }^{27}$ The Theory of Planned Behaviour is a psychosocial model that has been effectively applied to the prediction of many health behaviours by considering an individual's attitude, subjective norms and perceived behavioural control. ${ }^{27}$ Within Canada, alcohol is generally recognised as a socially acceptable and popular substance. ${ }^{1}$ Research suggests normative social influences and cultural norms may also play an important role in explaining drinking patterns. ${ }^{28}{ }^{29}$ Given this, behaviours may result from interactions with surrounding social environments and contribute to the development of positive perceptions of drinking. ${ }^{30-32}$ These positive perceptions can create an environment where drinking behaviour is both accepted and encouraged socially, and may contribute to increased alcohol consumption and binge drinking. ${ }^{30} 33$ Despite the potential hazardous health outcomes and biopsychosocial problems associated with binge drinking, ${ }^{3}$ it is often viewed as a positive social activity; an important aspect to consider when exploring the relationship between drinking and mental well-being. One study found that drinking with friends is protective against alcohol-related problems (eg, physical fights or injuries, driving under the influence, hangovers or vomiting). ${ }^{35}$ As such, research has suggested that social drinking may coincide with aspects of positive psychosocial well-being such as social participation, enhanced self-esteem and autonomy, coping strategies and accountability. ${ }^{36}$

Flourishing is the presence of positive mental health, inclusive of emotional, psychological, and social prosperity, and often used as an indicator of overall psychosocial well-being. ${ }^{37-39}$ Flourishing incorporates contemporary theories of well-being, acknowledging the importance of social relationships, not just for pleasure but self-perceived interest and engagement, and meaning and purpose in life. Given that flourishing represents a modernised concept in the field of psychology, limited research currently exists exploring the impact positive well-being may have on overall substance use behaviours. However, recent findings examining the association between mental health problems and cannabis use demonstrated that after controlling for depression and anxiety, youth who reported flourishing were found to be less likely to have used cannabis and less likely to have used at higher frequencies. ${ }^{40}$ As flourishing may offer protective effects against cannabis, ${ }^{40}$ additional research on the influence of overall well-being within other substance use domains (eg, alcohol and tobacco use) is necessary to inform strategies for youth prevention or intervention programming.

The authors are unaware of existing research that has evaluated indicators of mental well-being, such as flourishing, as a protective factor against binge drinking and mental health problems among youth. In response to this gap, the objective of the current study was to examine the association between binge drinking and depression and anxiety symptoms among a large sample of Canadian youth. Our analysis is intended as a follow-up to novel findings between cannabis and flourishing ${ }^{40}$ and we aim to contribute to the literature by examining whether flourishing moderates the association between depression, anxiety, and binge drinking behaviour within Canadian secondary school students.

\section{METHODS}

The COMPASS (Cannabis, Obesity, Mental health, Physical activity, Alcohol, Smoking, Sedentary Behaviour) Study is a prospective cohort study (2012-2021) that collects data from full school samples of students in grades 9 through 12 attending participating secondary schools across Canada. ${ }^{41}$ A new COMPASS mental health module (MH-M) was piloted in year 5 COMPASS data collections $\left(\mathrm{Y}_{5}(2016-2017)\right)$, as students' mental health 
was identified by stakeholders as a priority area for study. ${ }^{42}$ This paper uses the data collected from students attending the 14 COMPASS schools that were selected to participate in the MH-M pilot via the COMPASS student questionnaire. ${ }^{42}$ The original COMPASS study protocol was maintained with the exception of the eight new subsections about youth mental health that were added to supplement the original student questionnaire. ${ }^{41} 4344$ A complete description of COMPASS methods is available in print ${ }^{41}$ or online (www.compass.uwaterloo.ca). This study received ethics approval from the University of Waterloo Human Research Ethics Committee and all participating school boards.

Data were collected from 8344 students attending selected secondary schools in British Columbia $(n=5)$ and Ontario ( $n=9$ ), Canada. Specific schools were recruited to participate in the pilot stage of the mental health module during year 5 of the COMPASS data collection based on expressed interest in the mental health data. Students were recruited using a parental active-information passive-consent permission protocol, ${ }^{41}$ a strategy shown to be important for collecting robust data on self-reported risk behaviours such as substance use among youth. ${ }^{45-47}$ A complete-case analysis was used for this manuscript $(\mathrm{n}=8344)$, resulting in a final analytic sample of 6570 students.

\section{Patient and public involvement}

There were no patients involved in the development of the research study. School board stakeholders were involved throughout the development of the pilot mental health module as part of the working research team. Anonymised school-level, board-level and province-level results were prepared for applicable stakeholders in the form of a report. See www.compass.uwaterloo.ca for protocol details.

\section{MEASURES}

\section{Binge drinking}

Consistent with previous research, ${ }^{48}$ binge drinking was assessed by asking students 'In the last 12 months, how often did you have 5 drinks of alcohol or more on one occasion?' Responses were recoded into a binary measure of binge drinking, where students who indicated not having done this in the past 12 months, were classified as 'non-current binge drinkers' and all other responses were coded as 'current binge drinkers.' For ordinal responses, binge drinking frequency was collapsed into the following: 'non-current binge drinkers', consistent with the binary variable explained above; 'rare/sporadic', if respondents indicated binge drinking less than once a month; 'monthly', if reported use was once to three times per month, and; 'weekly,' if use ranged from once a week to daily.

\section{Mental health variables}

Youth depressive symptoms were assessed using the Center for Epidemiologic Studies Depression Scale (Revised)-10 $\left(\right.$ CESD-R- $\left.10^{49}\right)$. This 10 -item scale was designed to assess self-reported symptoms characteristic of depression such as feelings of sadness, hopelessness, apathy and motivation, irritability, and difficulties sleeping, making decisions, and concentrating over a 1 week period. ${ }^{49-51}$ Internal consistency of the CESD-R-10 scale was high $(\alpha=0.98)$. Anxiety symptoms were measured using the Generalised Anxiety Disorder 7-item Scale (GAD- $\left.7^{52}\right)$. The GAD-7 assesses on difficulty controlling feelings of worry, trouble relaxing, nervousness, restlessness and irritability over a 2 week period of time and had high internal consistency $(\alpha=0.99)$. Both scales have been validated for use in adolescent populations. ${ }^{50-52}$ The CESD-R-10 and GAD-7 scales were fit as dichotomous variables for each model; consistent with other research, this study applied a binary coding system to categorise students with and without clinically-relevant symptoms (control $=0$, depression and/ or anxiety=1) using scores $\geq 10$ for both the CESD-R- $10^{49} 51$ and GAD $-7^{52}$ to indicate risk of or probable depression and anxiety, respectively.

Levels of self-perceived psychosocial well-being among students was measured using Diener's Flourishing Scale $\left(F S^{53}\right)$. This scale provides a score that represents overall psychological functioning on a flourishing-languishing continuum by assessing how students perceive their: relationships, life purpose and satisfaction, engagement with and interest in daily activities, self-esteem, competence, and optimism. To remain suitable for large, school-based studies, ${ }^{44}$ the original 7-point Likert scale was reduced to a 5-point response option with total scores ranging from 8 to 40 . All item statements are positively framed within the original FS. ${ }^{53}$ However, to remain consistent with the other COMPASS MH-M measures, the FS was reverse coded where low scores represented flourishing (good overall well-being) and high scores represent languishing (an indication of poor overall psychosocial well-being). Internal consistency of the FS was high $(\alpha=0.98)$.

\section{Covariates}

The following covariates were accounted for: $\operatorname{grade}^{9-12}$; sex (female, male); ethnicity (White, Black, Asian, Indigenous (First Nations, Métis, Inuit)], Latin American or Hispanic, Mixed/Other); weekly spending money (\$C0, \$C1-20, \$C21-100, more than \$C100, I don't know; as a proxy for socioeconomic status, as this is a more accessible value for youth to report on than household income); truancy (no skipped classes, one or more missed classes per week); cannabis use (never use, ever user (ever using marijuana)); smoking status (non-smoker (reported never smoking or non-current use of cigarettes), current smoker (reported smoking one or more cigarettes in the past month)) and team sport involvement (not involved, involved (school- or community-level)).

\section{Analysis}

Among our analytic sample, descriptive analyses were conducted using $\chi^{2}$ and t-tests. Binge drinking status (non-current vs current binge drinker) was modelled 
using a binary logistic regression (model 1) and binge drinking frequency was modelled using an ordinal logistic regression (model 2) which adjusted for relevant covariates. Given the large sample size, a CI of $99 \%$ was used. The frequency of binge drinking was modelled to examine if mental health problems were associated with increasing levels of binge drinking frequencies. To examine flourishing as a moderating variable, 2-way and 3-way interactions between flourishing, depression and anxiety were tested. Only complete-case analysis was conducted where $11 \%$ of GAD-7 data, $18 \%$ of CESD-R-10 data and less than $1 \%$ of binge drinking was missing. The impact of data was assessed and we found no meaningful difference among students with and without missing data. All analyses were conducted in SAS V.9.4. ${ }^{54}$

\section{RESULTS}

\section{Sample characteristics}

Demographic characteristics for the total sample $(\mathrm{n}=6570)$ are presented in table 1 . The majority of students reported their ethnicity as white $(71.4 \%)$ and $51.6 \%$ were female. Overall, $37.0 \%$ of the sample reported binge drinking at least once within the past year and 20\% indicated binge drinking at least once a month. Clinically-relevant symptoms (scores $\geq 10$ ) for depression were reported by $41.4 \%$ of students in the sample and $31.7 \%$ reported symptoms of anxiety above the threshold (scores $\geq 10$ ). The mean flourishing score was 16.66 (SD:5.87) and after examining descriptive results by sex, females (17.23 (SD:6.00)) reported greater languishing scores than males (15.99 (SD:5.65)). More females reported clinically-relevant symptoms of depression (51.9\% vs $30.1 \%$ ) and anxiety $(43.5 \%$ vs $19.1 \%)$, compared with their male counterparts. Although the rate of binge drinking among females and males was similar, females were more likely to engage in sporadic $(18.7 \%$ vs $15.3 \%)$ and monthly $(15.2 \%$ vs $14.6 \%)$ binge drinking and less likely to binge drink weekly (3.9\% vs $6.3 \%)$, compared with males.

\section{Correlates of drinking behaviours}

Table 2 demonstrates results of the regression models used for predicting binge drinking behaviours. Youth reporting current binge drinking were more likely to be in grade 12 (adjusted OR (AOR) 2.31, (99\% CI 1.74 to 3.02 )), grade 11 (AOR 1.69, (99\% CI 1.29 to 2.20 )), and grade 10 (AOR 1.56, (99\% CI 1.21 to 2.00)) in Model 1. The same demographic trends were observed in Model 2 for binge drinking frequency, whereby students in older grades were more likely to report higher binge drinking frequencies. As seen in Model 1, cannabis use (AOR: 7.76, (99\% CI 6.36 to 9.46)), tobacco smoking (AOR 3.00, (99\% CI 2.12 to 4.25$)$ ), truancy (AOR 2.29, (99\% CI 1.90 to 2.76) ) and being involved with a sports team (AOR 1.67, (99\% CI 1.37 to 2.03$)$ ) were significantly associated with an increased odds of binge drinking. Similarly, cannabis use (AOR 7.12, (99\% CI 5.95 to 8.52)), tobacco smoking (AOR 3.94, (99\% CI 3.11 to 4.99)), truancy (AOR 2.04,
(99\% CI 1.73 to 2.41$)$ ) and being involved on a sports team (AOR 1.64, (99\% CI 1.39 to 1.93)) were associated with increases in binge drinking frequency in Model 2. Compared with those who reported not having any spending money, youth with \$C1-20 of weekly spending money were 1.37 times more likely to binge brink and the odds of binge drinking doubled (AOR: 2.68) for youth with greater than $\$ \mathrm{C} 100$ of weekly spending money (Model 1). Although mental health indicators were not found to be statistically significant, directionality of the AORs suggests a potentially positive association between depression and binge drinking (AOR: 1.98, (99\% CI 0.76 to 5.13)), as well as a negative association between anxiety and binge drinking (AOR: 0.57, (99\% CI 0.15 to 2.22)).

\section{Moderating effects of flourishing}

Above-threshold symptoms of depression and anxiety, and self-reported flourishing were not found to be significantly associated with binge drinking or binge drinking frequency within our sample. Moreover, the addition of interaction terms did not lead to any changes in estimations or significance; therefore, flourishing did not moderate the association between depression or anxiety symptoms and binge drinking behaviours as hypothesised.

\section{DISCUSSION}

Our findings correspond with previous surveillance research and national averages showing alcohol is the most common substance used among our sample of Canadian adolescents. ${ }^{12}$ This study identified that more than one-third of youth in Ontario and British Columbia, Canada who participated in the COMPASS MH-M pilot study, reported binge drinking within the past 12 months, and almost one in six students reported this behaviour monthly. Within our large sample of youth, nearly one-half of students indicated symptoms of clinically-relevant depression and one-third reported clinically-relevant symptoms of anxiety. Consistent with other research ${ }^{55}$ as well as existing findings from the COMPASS MH-M pilot study, ${ }^{40}$ neither depression nor anxiety were correlated with binge drinking. However, unlike previous COMPASS research that found flourishing may protect against cannabis use, ${ }^{40}$ these corresponding associations were not observed within this study for binge drinking. Based on these findings, youth may use alcohol for different reasons than cannabis, suggesting the two substances may play distinct social and psychological roles.

As literature continues to present inconsistent findings between mental health and substance use, it is critical to acknowledge subgroups of consumers ${ }^{18} 56$ as underlying motives for alcohol consumption may represent important insight for intervention strategies. ${ }^{57} \mathrm{In}$ Canada, alcohol consumption exists as a normative social behaviour ${ }^{58}$ that is common to many populations, ${ }^{1}$ including youth..$^{28} 29$ This custom may provide reason for the lack of associations observed in this study, and suggests that alcohol consumption may more commonly 
Table 1 Sample descriptives by sex for grade 9-12 students in year 5 of the mental health pilot of COMPASS (2016-2017)

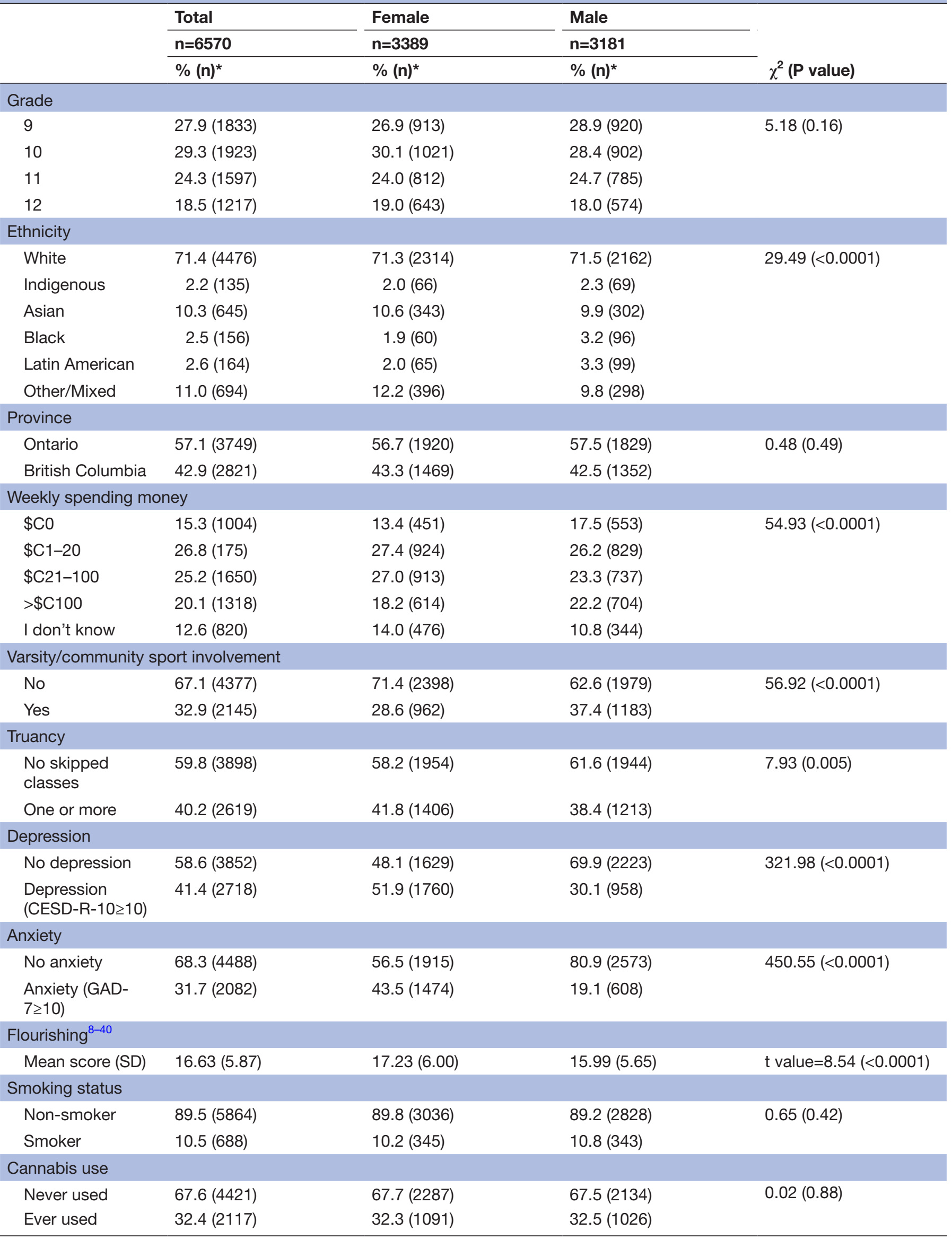


Table 1 Continued

\begin{tabular}{|c|c|c|c|c|}
\hline & Total & Female & Male & \multirow[b]{3}{*}{$\chi^{2}$ (P value) } \\
\hline & $n=6570$ & $n=3389$ & $n=3181$ & \\
\hline & $\%(n)^{\star}$ & $\%(n)^{*}$ & $\%(n)^{\star}$ & \\
\hline $\begin{array}{l}\text { Non-current binge } \\
\text { drinker }\end{array}$ & $63.0(4137)$ & $62.2(2109)$ & 63.7 (2028) & $1.63(0.20)$ \\
\hline $\begin{array}{l}\text { Non-current binge } \\
\text { drinker }\end{array}$ & $63.0(4137)$ & $62.2(2109)$ & $63.8(2028)$ & \multirow[t]{3}{*}{$29.27(<0.0001)$} \\
\hline Rare/sporadic & $17.0(1120)$ & $18.7(631)$ & $15.3(489)$ & \\
\hline Monthly & $14.9(980)$ & $15.2(516)$ & $14.6(464)$ & \\
\hline
\end{tabular}

*Note: The numbers may not add up to the total due to missing values and rounding. Complete case analysis was conducted; $11 \%$ of GAD-7 data, $18 \%$ of CESD-R-10 data, and less than $1 \%$ of binge drinking data was missing.

CESD-R-10, Center for Epidemiologic Studies Depression Scale (Revised)-10; GAD-7, Generalised Anxiety Disorder 7-item Scale.

occur as a social activity rather than a coping mechanism for negative affect, attenuating potential associations between mental health problems and binge drinking. Such links between social influence and binge drinking have also been demonstrated elsewhere ${ }^{59-62}$ and ultimately provide support for the Theory of Planned Behaviour. Schlegel $e t a l^{62}$ found attitudes towards binge drinking and perceived social norms all contributed to the intention and frequency of binge drinking. Moreover, binge drinking in youth has been shown to be associated with peer group influences, whereby peer alcohol use may be the strongest predictor of adolescent drinking behaviour, presenting important implications for intervention approaches. ${ }^{28}{ }^{63}$ Distal components of this theory, such as socioeconomic status, were also found to be associated with binge drinking in our sample. We identified a strong dose-response relationship between binge drinking and weekly spending money, with greater amounts of weekly spending money predicting an increased likelihood of binge drinking and binge drinking frequency. Our results may reflect a correlation between socioeconomic status and alcohol consumption among youth, or at least appear to indicate that available spending money (eg, provided by parental, part-time employment or other sources) provides the means to purchase alcohol.

These findings supplement other substance use literature and identify that co-occurring substance use is common among students who binge drink. ${ }^{64-67}$ Youth engaging in current binge drinking were approximately three times more likely to smoke tobacco and almost eight times more likely to use cannabis. Sports team participation appeared to also be a strong predictor of binge drinking. Adding this variable improved the predictive accuracy of our logistic models, as demonstrated by the concordance statistic. While sports team participation can contribute to positive health behaviours among youth (eg, physical activity), patterns of excessive alcohol consumption among school athletes has been previously observed in research. ${ }^{68}$

The Canadian Centre on Substance Abuse recognises that sports participation is linked to decreases in illicit drug use among youth, but the use of alcohol increases. ${ }^{69}$ Youth between the ages of 15 and 19 years have the highest participation rates in sports. ${ }^{70}$ Athletic involvement presents an optimal opportunity to influence youth by leveraging their team sport environments and implementing targeted prevention strategies. ${ }^{69}$ Substance use is commonly initiated during adolescence, where enrolment in sports is high, and as such, this is a critical period for substance use prevention efforts. ${ }^{71}$ For instance, public health authorities may consider limiting or restricting alcohol promotion during events that may be attended by youth. Continued research is necessary within this domain as there remains a paucity of evidence on the risk of binge drinking within a team dynamic.

Given the varied predictors for alcohol and cannabis use among youth in the COMPASS MH-M pilot study, tailored approaches for each individual substance may be necessary. Although statistically insignificant, our findings present interesting results that suggest depression and anxiety may have different outcomes for alcohol consumption, and the directionality of associations observed may provide insight to public health researchers. Based heavily on the Theory of Planned Behaviour, existing psychosocial research suggests that persuading attitudes and norms to influence behaviour may prove effective at producing long-term changes. ${ }^{72}$ Successful strategies may involve peer-to-peer mentoring or social norming campaigns given the influence peer groups have on attitudes towards alcohol consumption and, in turn, binge drinking behaviour. ${ }^{28} 2932$ Also, targeted intervention efforts implemented within the environment of 
Table 2 Binge drinking for grade 9-12 students in year 5 of the COMPASS MH-M (2016-2017)

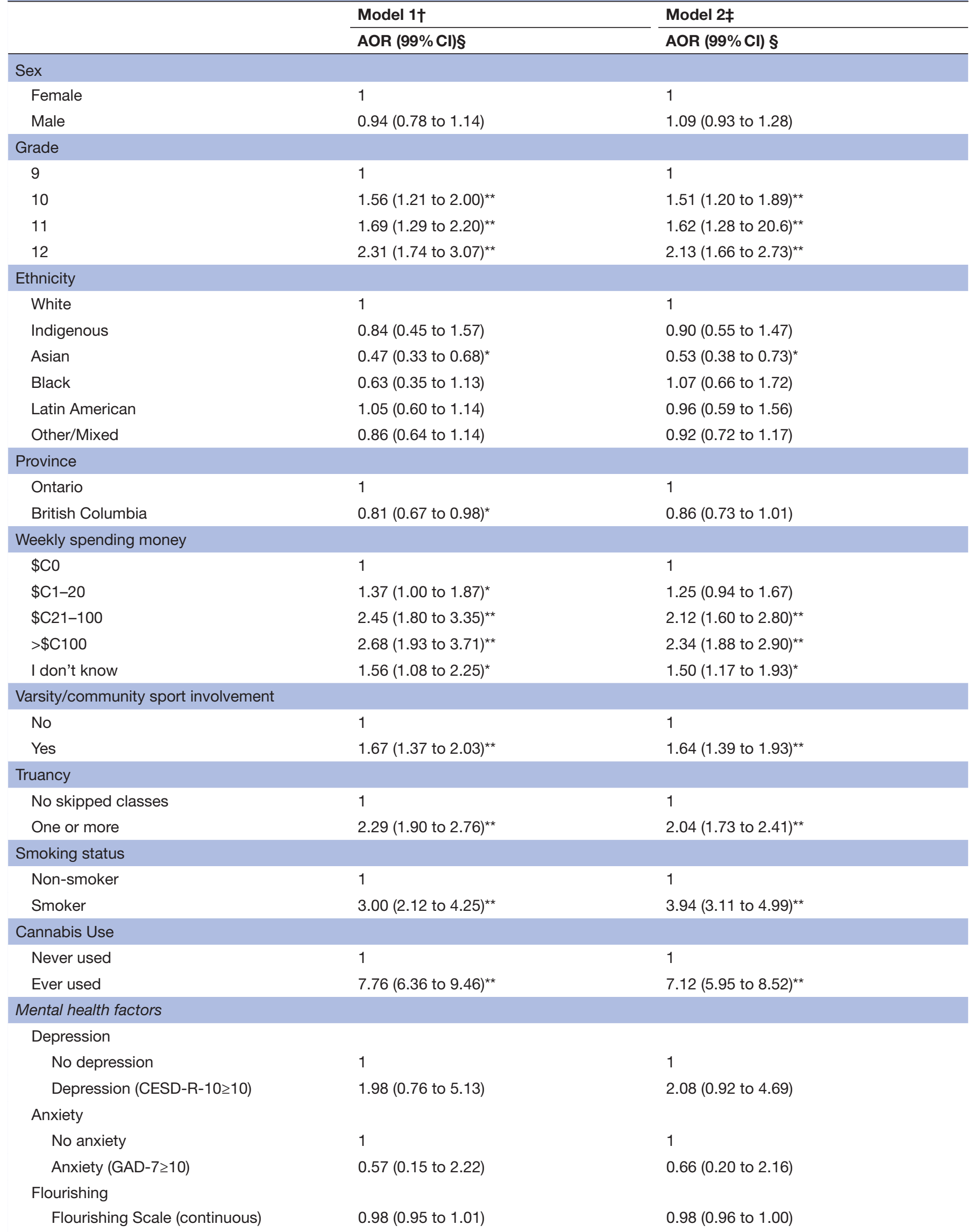




\begin{tabular}{|c|c|c|}
\hline & Model 1† & Model 2‡ \\
\hline & AOR $(99 \% \mathrm{Cl}) \S$ & AOR $(99 \% \mathrm{Cl}) \S$ \\
\hline \multicolumn{3}{|l|}{ Interactions } \\
\hline Depression^flourishing & 0.98 (0.92 to 1.03$)$ & 0.97 (0.92 to 1.01$)$ \\
\hline Anxiety*flourishing & 1.05 (0.96 to 1.14$)$ & 1.04 (0.97 to 1.13$)$ \\
\hline Concordance statistic & 0.862 & 0.836 \\
\hline
\end{tabular}

${ }^{*} \mathrm{p} \leq 0.01,{ }^{* *} \mathrm{p}<0.001$.

†Note: model 1 is a logistic regression of mental health predictors and binge drinking status.

$\ddagger$ Note: model 2 is an ordinal logistic regression of mental health predictors and binge drinking frequency.

§Note: complete case analysis was conducted and 2395 observations were deleted due to missing data for the mental health and binge drinking variables.

AOR, adjusted OR; CESD-R-10, Center for Epidemiologic Studies Depression Scale (Revised)-10; GAD-7, Generalised Anxiety Disorder 7-item Scale; MH-M, mental health module.

a sports team dynamic may effectively influence health behaviours. Research should aim to examine substance use behaviours both separately and concurrently; further exploration may consider the possible association between mental health and co-occurring alcohol and cannabis use. Future waves of the COMPASS study will be able to test these correlations and temporal relationships.

\section{Strengths and limitations}

The COMPASS study is a unique data system that examines Canadian youth risk behaviours, and provides an ideal platform to evaluate multiple co-occurring behaviours and changes over time. Our results supplement existing evidence and advance knowledge on youth binge drinking behaviours. Important correlates identified in this study can be used to inform the creation of effective prevention and intervention programmes. While this study provides a novel contribution to the literature, it is not without limitations. COMPASS uses a purposeful sampling method, and results are not representative of all Ontario and British Columbia youth or secondary schools. Since student data are self-reported, findings may be subject to reporting or recall bias, however, passive permission protocols have been implemented to improve sample representativeness and preserve anonymity. By employing a complete-case analysis, associations observed within this study may be underestimated. Data appeared to missing at random and information bias from non-differential misclassification is possible. Mental health items were measured based on 1-2-week recall and although scales employed in this study have been widely validated, these measures cannot determine whether symptoms were chronic or acute. Moreover, depression and anxiety data were measured using scales and not diagnostic criteria. This study was unable to examine broader social environments (eg, social acceptability of alcohol, sport team dynamics) that may be associated with alcohol consumption patterns. Lastly, we were unable to establish binge drinking trajectories in relation to depression and anxiety given the cross-sectional nature of the data. As COMPASS employs a longitudinal study design, follow-up research and examination of how behaviours evolve over time is possible once future waves of mental health data become available.

\section{CONCLUSION}

A large proportion of the study sample of grade 9 to 12 students reported binge drinking. About one-half and one-third of students reports clinically-relevant symptoms of depression and anxiety respectively, however, our research shows that mental health problems were likely not responsible for explaining patterns of alcohol consumption in our youth sample. Contrary to other COMPASS research on cannabis use, flourishing was not shown to moderate the relationship between depression or anxiety and binge drinking in secondary school students. This new analysis of the COMPASS mental health data and youth alcohol use suggests that binge drinking among youth may be a product of social acceptability and normalcy, as opposed to a coping mechanism or contributor to mental health problems. Future research should aim to consider the role of social environments and alcohol consumption among youth, and where data is available, targeting binge drinking within a team dynamic and among different types of sports.

Contributors $A B$ and IR conceived the manuscript idea, performed the statistical analyses, drafted the manuscript and revised the manuscript for content. IR, MAF, $\mathrm{KP}, \mathrm{MdG}$, and YJ revised the manuscript for critical content. STL conceived the host study, led the acquisition of all data, drafted components of introduction and discussion, and revised the manuscript for critical content. All authors who have contributed significantly to the work presented within this manuscript have been listed above.

Funding The COMPASS study has been supported by a bridge grant from the CIHR Institute of Nutrition, Metabolism and Diabetes (INMD) through the "Obesity - Interventions to Prevent or Treat" priority funding awards (00P-110788; grant awarded to STL), an operating grant from the CIHR Institute of Population and Public Health (IPPH) (MOP-114875; grant awarded to STL), a CIHR Project Grant (PJT148562; grant awarded to STL), a CIHR Project Grant (PJT-149092; grant awarded 
to KP), and by a research funding arrangement with Health Canada (\#1617-HQ000012; contract awarded to STL).

Competing interests None declared.

Patient consent for publication Not required

Ethics approval The COMPASS Study received ethics approval from the University of Waterloo Office of Research Ethics as well as participating school boards (ORE \#: 30118).

Provenance and peer review Not commissioned; externally peer reviewed.

Data sharing statement $A$ data request form to access to the COMPASS data can be found at: https://uwaterloo.ca/compass-system/information-researchers/datausage-application

Open access This is an open access article distributed in accordance with the Creative Commons Attribution Non Commercial (CC BY-NC 4.0) license, which permits others to distribute, remix, adapt, build upon this work non-commercially, and license their derivative works on different terms, provided the original work is properly cited, appropriate credit is given, any changes made indicated, and the use is non-commercial. See: http://creativecommons.org/licenses/by-nc/4.0/.

\section{REFERENCES}

1. Public Health Agency of Canada. The Chief Public Health Officer's Report on the State of Public Health in Canada 2018: Preventing Problematic Substance Use in Youth [Internet]. Ottawa 2018. https://www.canada.ca/en/public-health/corporate/publications/ chief-public-health-officer-reports-state-public-health-canada/2018preventing-problematic-substance-use-youth.html.

2. Canada H. Summary of results for the Canadian Student Tobacco, Alcohol and Drugs Survey 2016-17 [Internet]. 2018 https://www. canada.ca/en/health-canada/services/canadian-student-tobaccoalcohol-drugs-survey/2016-2017-summary.html.

3. Welch KA, Carson A, Lawrie SM. Brain structure in adolescents and young adults with alcohol problems: systematic review of imaging studies. Alcohol Alcohol 2013;48:433-44.

4. Squeglia LM, Tapert SF, Sullivan EV, et al. Brain development in heavy-drinking adolescents. Am J Psychiatry 2015;172:531-42.

5. Hanson KL, Medina KL, Padula CB, et al. Impact of Adolescent Alcohol and Drug Use on Neuropsychological Functioning in Young Adulthood: 10-Year Outcomes. J Child Adolesc Subst Abuse 2011;20:135-54

6. Lo CC, Weber J, Cheng TC. A spatial analysis of student binge drinking, alcohol-outlet density, and social disadvantages. Am J Addict 2013;22:391-401.

7. Larsen $\mathrm{K}$, To T, Irving HM, et al. Smoking and binge-drinking among adolescents, Ontario, Canada: Does the school neighbourhood matter? Health Place 2017;47:108-14.

8. Campbell CA, Hahn RA, Elder R, et al. The effectiveness of limiting alcohol outlet density as a means of reducing excessive alcohol consumption and alcohol-related harms. Am J Prev Med 2009;37:556-69.

9. Wechsler H, Davenport A, Dowdall G, et al. Health and behavioral consequences of binge drinking in college. A national survey of students at 140 campuses. JAMA 1994;272:1672-7.

10. Miller JW, Naimi TS, Brewer RD, et al. Binge drinking and associated health risk behaviors among high school students. Pediatrics 2007; 119:76-85.

11. Marmorstein NR, lacono WG, Malone SM. Longitudinal associations between depression and substance dependence from adolescence through early adulthood. Drug Alcohol Depend 2010;107:154-60.

12. Hoel S, Eriksen BM, Breidablik H-J, et al. psychological health, and social integration. Scand J Public Health 2004.

13. Archie S, Zangeneh Kazemi A, Akhtar-Danesh N. Concurrent binge drinking and depression among Canadian youth: prevalence, patterns, and suicidality. Alcohol 2012;46:165-72.

14. Mason WA, Kosterman R, Haggerty KP, et al. Dimensions of adolescent alcohol involvement as predictors of young-adult major depression. J Stud Alcohol Drugs 2008;69:275-85.

15. Tapert SF, Caldwell L, Burke C. Alcohol and the adolescent brain: Human studies. Alcohol Res Heal [Internet] 2004 http://go.galegroup.

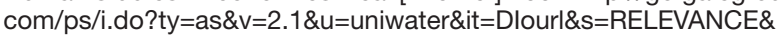
$\mathrm{p}=\mathrm{AONE} \& \mathrm{qt}=\mathrm{SP} \sim 205 \sim \sim \mathrm{IU} \sim 4 \sim \sim \mathrm{SN} \sim 1535-7414 \sim \sim \mathrm{VO} \sim 28 \& \mathrm{Im}=\mathrm{DA} \sim$ $120040000 \& s w=w$

16. Lamis DA, Malone PS, Langhinrichsen-Rohling J, et al. Body investment, depression, and alcohol use as risk factors for suicide proneness in college students. Crisis 2010;31:118-27.
17. Lansford JE, Erath S, Yu T, et al. The developmental course of illicit substance use from age 12 to 22: links with depressive, anxiety, and behavior disorders at age 18. J Child Psychol Psychiatry 2008;49:877-85.

18. Colder CR, Chassin L. The psychosocial characteristics of alcohol users versus problem users: data from a study of adolescents at risk. Dev Psychopathol 1999;11:321-48.

19. Dir AL, Bell RL, Adams ZW, et al. Gender Differences in Risk Factors for Adolescent Binge Drinking and Implications for Intervention and Prevention. Front Psychiatry 2017;8.

20. Bulloch AG, Williams JV, Lavorato $\mathrm{DH}$, et al. Trends in binge drinking in Canada from 1996 to 2013: a repeated cross-sectional analysis. CMAJ Open 2016;4:E599-E604.

21. Johnston LD, O'Malley PM, Bachman JG, et al. Monitoring the future National survey results on drug use, 1975-2010. Institute for Social Research 2011.

22. O'Hara RE, Cooper ML. Bidirectional associations between alcohol use and sexual risk-taking behavior from adolescence into young adulthood. Arch Sex Behav 2015;44:857-71.

23. Leatherdale ST. An examination of the co-occurrence of modifiable risk factors associated with chronic disease among youth in the COMPASS study. Cancer Causes Control 2015;26:519-28.

24. Kwan M, Bobko S, Faulkner G, et al. Sport participation and alcohol and illicit drug use in adolescents and young adults: a systematic review of longitudinal studies. Addict Behav 2014;39:497-506.

25. Bellis MA, Hughes $\mathrm{K}$, Morleo M, et al. Predictors of risky alcohol consumption in schoolchildren and their implications for preventing alcohol-related harm. Subst Abuse Treat Prev Policy 2007;2:15.

26. Chung T, Creswell KG, Bachrach R, et al. Adolescent binge drinking: Developmental context and opportunities for prevention. Alcohol Res Curr Rev 2018.

27. Ajzen I. The theory of planned behavior. Organ Behav Hum Decis Process 1991.

28. Brooks-Russell A, Simons-Morton B, Haynie D, et al. Longitudinal relationship between drinking with peers, descriptive norms, and adolescent alcohol use. Prev Sci 2014;15:497-505.

29. Jun M, Agley J, Huang C, et al. and Social Norms: Advancing Understanding Through Statistical Applications. J Child Adolesc Subst Abus 2016.

30. Tanski SE, McClure AC, Li Z, et al. Cued recall of alcohol advertising on television and underage drinking behavior. JAMA Pediatr 2015;169:264.

31. Hastings G, Anderson S, Cooke E, et al. Alcohol marketing and young people's drinking: a review of the research. J Public Health Policy 2005;26:296-311.

32. Ali MM, Amialchuk A, Nikaj S. Alcohol consumption and social network ties among adolescents: evidence from Add Health. Addict Behav 2014;39:918-22.

33. Smith LA, Foxcroft DR. The effect of alcohol advertising, marketing and portrayal on drinking behaviour in young people: systematic review of prospective cohort studies. BMC Public Health 2009;9:51.

34. Gordon R, MacKintosh AM, Moodie C. The impact of alcohol marketing on youth drinking behaviour: a two-stage cohort study. Alcohol Alcohol 2010;45:470-80.

35. Clapp JD, Shillington AM, Segars LB. Deconstructing contexts of binge drinking among college students. Am J Drug Alcohol Abuse 2000;26:139-54.

36. Nyqvist F, Pape B, Pellfolk T, et al. Structural and cognitive aspects of social capital and all-cause mortality: A meta-analysis of cohort studies. Soc Indic Res 2014.

37. Diener E, Wirtz D, Tov W, et al. New well-being measures: Short scales to assess flourishing and positive and negative feelings. Soc Indic Res 2010;97:143-56.

38. Silva AJ, Caetano A. Validation of the flourishing scale and scale of positive and negative experience in portugal. Soc Indic Res 2013;110:469-78.

39. Keyes CL. Mental illness and/or mental health? Investigating axioms of the complete state model of health. J Consult Clin Psychol 2005;73:539-48.

40. Butler A, Patte KA, Ferro MA, et al. Interrelationships among depression, anxiety, flourishing, and cannabis use in youth. Addict Behav 2019;89:206-15.

41. Leatherdale ST, Brown KS, Carson V, et al. The COMPASS study: a longitudinal hierarchical research platform for evaluating natural experiments related to changes in school-level programs, policies and built environment resources. BMC Public Health 2014;14:331.

42. Patte KA, Henderson J, Faulkner G, et al. Development of a mental health module for the COMPASS system: Improving youth mental health trajectories. Internet]. Waterloo 2017 www.compass. uwaterloo.ca. 
43. Patte KA, Bredin C, Henderson J, et al. Development of a mental health module for the COMPASS system: Improving youth mental health trajectories. Part 2: Pilot test and focus group results [Internet]. 2017 www.compass.uwaterloo.ca.

44. Patte KA, Bredin C, Henderson J, et al. Development of a mental health module for the COMPASS system: Improving youth mental health trajectories. Part 1: Draft Development and Design [Internet] 2017. www.compass.uwaterloo.ca

45. Flicker S, Guta A. Ethical approaches to adolescent participation in sexual health research. J Adolesc Health 2008;42:3-10.

46. White VM, Hill DJ, Effendi Y. How does active parental consent influence the findings of drug-use surveys in schools? Eval Rev 2004;28:246-60.

47. Hollmann CM, Mcnamara JR. Considerations in the use of active and passive parental consent procedures. J Psychol Interdiscip Appl 2010.

48. Elton-Marshall T, Leatherdale ST, Manske SR, et al. Research methods of the Youth Smoking Survey (YSS). Chronic Dis Inj Can 2011;32:47-54

49. Andresen EM, Malmgren JA, Carter WB, et al. Screening for depression in well older adults: evaluation of a short form of the CES-D (Center for Epidemiologic Studies Depression Scale). Am J Prev Med 1994;10:77-84.

50. Zhang W, O'Brien N, Forrest Jl, et al. Validating a shortened depression scale (10 item CES-D) among HIV-positive people in British Columbia, Canada. PLoS One 2012;7:e40793.

51. Bradley KL, Bagnell AL, Brannen CL. Factorial validity of the center for epidemiological studies depression 10 in adolescents. Issues Ment Health Nurs 2010;31:408-12.

52. Spitzer RL, Kroenke K, Williams JB, et al. A brief measure for assessing generalized anxiety disorder: the GAD-7. Arch Intern Med 2006;166:1092

53. Diener E, Wirtz D, Tov W, et al. New well-being measures: Short scales to assess flourishing and positive and negative feelings. Soc Indic Res 2010.

54. SAS Institute. SAS version 9.4. SAS Institute Inc, 2012.

55. Low KG. Flourishing, substance use, and engagement in students entering college: a preliminary study. J Am Coll Health 2011;59:555-61.

56. Chassin L, Pitts SC, Prost J. Binge drinking trajectories from adolescence to emerging adulthood in a high-risk sample: predictors and substance abuse outcomes. J Consult Clin Psychol 2002;70:67-78.

57. Cox WM, Klinger E. A motivational model of alcohol use. J Abnorm Psychol 1988;97:168-80.
58. Social Issues Research Centre. Social and cultural aspects of drinking [Internet]. 1998 http://www.sirc.org/publik/social_drinking. pdf.

59. Cooke R, Dahdah M, Norman P, et al. How well does the theory of planned behaviour predict alcohol consumption? A systematic review and meta-analysis. Health Psychol Rev 2016;10:148-67.

60. Norman P, Bennett $\mathrm{P}$, Lewis $\mathrm{H}$. Understanding binge drinking among young people: an application of the Theory of Planned Behaviour. Health Educ Res 1998;13:163-9.

61. Johnston KL, White KM. Binge-drinking: A test of the role of group norms in the theory of planned behaviour. Psychol Health 2003.

62. Schlegel RP, DAvernas JR, Zanna MP, et al. Problem drinking: A problem for the theory of reasoned action? J Appl Soc Psychol 1992.

63. Trucco EM, Colder CR, Wieczorek WF. Vulnerability to peer influence: a moderated mediation study of early adolescent alcohol use initiation. Addict Behav 2011;36:729-36.

64. Patton GC, Hibbert M, Rosier MJ, et al. Patterns of common drug use in teenagers. Aust J Public Health 1995;19:393-9.

65. Bailey SL. Adolescents' multisubstance use patterns: the role of heavy alcohol and cigarette use. Am J Public Health 1992;82:1220-4.

66. Yamaguchi K, Kandel DB. Patterns of drug use from adolescence to young adulthood: III. Predictors of progression. Am J Public Health 1984;74:673-81.

67. Everett SA, Giovino GA, Warren CW, et al. Other substance use among high school students who use tobacco. J Adolesc Health 1998;23:289-96.

68. Carr S, O'Brien KS, Ferris J, et al. Child and adolescent exposure to alcohol advertising in Australia's major televised sports. Drug Alcohol Rev 2016;35:406-11.

69. McKiernan A. Youth sport programs that address substance use - an environmental scan [Internet]. 2016 http://www.ccdus.ca/ Resource Library/CCSA-Sport-Programs-Substance-Use-EducationReport-2016-en.pdf.

70. Statistics Canada. Sport participation 2010 [Internet]. 2013 http:// publications.gc.ca/collections/collection_2013/pc-ch/CH24-1-2012eng.pdf.

71. Office of Research and Surveillance. Canadian Tobacco Alcohol and Drugs (CTADS): 2013 summary [Internet]. CTADS 2015;2013 https:// www.canada.ca/en/health-canada/services/canadian-tobaccoalcohol-drugs-survey/2013-summary.html.

72. Ajzen I. Attitudes and Persuasion: In. The Oxford Handbook of Personality and Social Psychology, 2012. 